LEschIK, G.: Mikrobotanisch-stratigraphische Untersuchungen in der Jungpliozänen Braunkohle von Buchenau (Kr. Hünefeld). - Paläontographica 92, Abt. B. Stuttgart 1951.

Lona, F.: Contributi alle storia della vegetazione e del clima nella val Pagana. Analisi pollinica del giacimento Villafranchiano di Leffe (Bergamo). - Mailand 1950.

Pflug, H.: Die stratigraphische Gliederung des kontinentalen Alttertiärs auf mikropaläontologischer (palynologischer u. a.) und kohlenpetrographischer Grundlage. - Diss. Archiv d. Univ. Bonn 1951. - Die Stratigraphie des eozänen Braunkohlenlagers von Helmstedt auf mikropaläontologischer Grundlage. Als Manuskript im Archiv des Amtes f. Bodenforschung. Krefeld 1950.

ReIN, U.: Pollenanalytische Untersuchungen zur Pliozän-Pleistozängrenze am linken Niederrhein. - Geol. Jahrb. 65. Hannover/Celle 1950.

Thomson, P. W.: Grundsätzliches zur tertiären Pollen- und Sporenmikrostratigraphie auf Grund einer Untersuchung des Hauptflözes der Rheinischen Braunkohle in Liblar/Bz. Köln. - Geol. Jahrb. 65. Hannover/Celle 1950. - Die Entstehung von Kohlenflözen auf Grund von mikropaläontologischen Untersuchungen des Hauptflözes der Rheinischen Braunkohle. - Zeitschr. f. Braunkohle, Wärme u. Energie, H. 3/4. Düsseldorf 1950.

Thomson P. W. \& Grebe, H.: Die Gliederung des Deckgebirges der Rheinischen Braunkohle. - Zeitschr. f. Braunkohle, Wärme u. Energie, H. 4, Düsseldorf 1951.

Venzo, S.: Rinvenimento di Anancus arvernensis nel Villafranchiano dell' Adda 'di Paderno, di Archidiskodon meridionalis e Cervus a Leffe. Stratigrafia e clima del Villafrachiano bergamasco. - Mailand 1950.

Wirtz, D.: Die Fauna des Sylter Crag und ihre Stellung im Neogen der Nordsee. Mitt. Geol. Staatsdienst Hamburg, H. 19. 1949.

Wolters, R.: Nachweis der Günzeiszeit und der Günz-Mindel-Wärmezwischenzeit am Niederrhein. - Geol. Jahrb. 65. Hannover/Celle 1950.

\title{
Zur Vegetationsentwicklung des Interglazials von Großweil ${ }^{1}$ )
}

\author{
Von Helga Reich. Mit $1 \mathrm{Abb}$.
}

Aus den nördlichen Alpen und ihrem Vorland sind zahlreiche pflanzenführende Interglaziale bekannt, vor allem stark zusammengepreßte interglaziale Torfe, die als "Schieferkohlen“ bezeichnet werden. Der Pflanzenbestand dieser Schieferkohlen ist erstaunlich einförmig. In den Pollendiagrammen herrschen Kiefer und Fichte weitaus vor. Nur zeitweise werden Tanne, Erle oder Hasel häufig. Die übrigen Gehölze sind äußerst spärlich, die Rotbuche (Fagus) fehlt an den meisten Fundorten völlig (vgl. LüDi 1946). Danach enthalten die Schieferkohlen nur Teile der interglazialen Vegetationsentwicklung. Diese in Pollendiagrammen vollständig zu erfassen, ist bisher nicht gelungen.

Die Gleichförmigkeit der Flora der Schieferkohlen macht es sehr schwierig, aus dem Pflanzenbestand Anhaltspunkte zur Bestimmung ihres Alters und ihrer Stellung innerhalb eines Interglazials zu gewinnen. Die starke Ausbreitung der Fichte, die in den weiter nördlich gelegenen Gebieten vor allem für die späteren Abschnitte der beiden letzten Interglaziale bezeichnend ist, spricht für das Ende eines Interglazials. Da es sich bei den Schieferkohlen in erster Linie um Versumpfungsmoore handelt, gewinnt man den Eindruck, daß im Alpenbereich erst die Niederschlagszunahme und Temperaturabnahme des ausklingenden Interglazials eine ausgedehnte Vermoorung der Tieflagen ermöglicht hat (Firbas 1951).

Bei einer erneuten Untersuchung der Schieferkohlen von Großweil ist es nun gelungen, die Vegetationsentwicklung dieses Interglazials nahezu vollständig zu

1) Vorläufige Mitteilung; aus dem Systematisch-Geobotanischen Institut der Universität. Göttingen. 
erfassen. Eine eingehende Darstellung der Untersuchungsergebnisse wird nach ihrem Abschluß an anderer Stelle erfolgen. Hier sei ein vorläufiger Bericht über die Hauptzüge der Vegetationsentwicklung vorausgeschickt.

Großweil liegt am Rande des Kochelseebeckens, zwischen Flyschbergen und Molassezone des Voralpenlandes. Die Schieferkohlen, die dort abgebaut werden, werden nach geologischen Befunden ins letzte, also Riß-Würm-Interglazial gestellt (vgl. Troll 1937). Über einige in Großweil gefundene Pflanzenreste hat Schuster (1909) berichtet. Zwei von mir untersuchte Profile aus den beiden dort aufgeschlossenen Flözen, die durch ein Tonband getrennt sind, ergaben zunächst nur Diagramme, die mit ihrem offenbar gesetzmäßigen Wechsel von Fichtenund Kieferngipfeln dem gewohnten Bild der Schieferkohlen entsprechen. Lediglich in den untersten Proben treten mit größerer Häufigkeit Tanne, Hainbuche und andere Gattungen auf. Schon damit wurde sehr wahrscheinlich, daß der Bildung der ausgedehnten Schieferkohlenmoore mit wechselnden Kiefern- und Fichtenphasen eine Zeit mit artenreicheren Mischwäldern, also wohl eine wärmere interglaziale Phase, vorausging. Ein im September 1951 unter dem Hauptflöz durch Grabung gewonnenes Profil von nur $65 \mathrm{~cm}$ Mächtigkeit, das teils aus humosen Tonen, teils aus Schieferkohle besteht, bestätigte diesen Schluß und ergänzte die Befunde aus den Kohlenflözen sogar bis in den Anfang des Interglazials zurück.

Aus den vorliegenden Diagrammen wurde ein halbschematisches Diagramm entworfen (Abb. 1), indem aus jedem bezeichnenden Abschnitt zwei Pollenspektren herausgegriffen und in der richtigen Reihenfolge, aber in gleichen Abständen eingetragen wurden. Die Ablagerung beginnt mit dem Ende einer Kiefernzeit und mit dem Beginn der Ausbreitung von Hasel, Eiche, Ulme und Erle. Gleichzeitig tritt aber bereits die Fichte auf und nimmt so stark zu, daß die folgende Periode als Fichten-Hasel-Eichenmischwaldzeit bezeichnet werden muß. Es muß vorläufig dahingestellt bleiben, ob diese im Vergleich zu den postglazialen Diagrammen der Loisach-Kochelsee-Moore (PAUL \& RuofF) und zu einem eigenen Diagramm von Sindelsdorf kräftigere Ausbreitung der Fichte ein für dieses Interglazial bezeichnender Zug ist oder auf örtlicher Förderung des Baumes beruht. Im Laufe der Fichten-Hasel-Eichenmischwaldzeit erscheinen in geringen Mengen Linde und Esche, in Spuren auch die Hainbuche, und gegen Ende erreicht die Hasel ihr absolutes Maximum (38\%). Auf die Fichten-Hasel-Eichenmischwaldzeit folgt weiter eine Erlen-Fichten-Hainbuchenphase, in der der Pollen schlecht erhalten ist und die Vorherrschaft der Erle örtlich bedingt sein dürfte. Wesentlich ist, daß sich während dieser Zeit die Hainbuche ausbreitet und häufiger wird als der Eichenmischwald. Um diese Zeit erscheinen die ersten Pollenkörner der Tanne (Abies), die während einer erneuten Zunahme der Fichte ihre relativen Höchstwerte erreicht $(28,5 \%)$. Man kann jetzt von einer Fichten(Erlen)-Tannenzeit sprechen. Um diese Zeit erst setzte in Großweil und dem benachbarten Ohlstadt die ausgedehnte Vermoorung ein, auf die die heutigen Schieferkohlenflöze zurückgehen. Auch andere nordalpine und voralpine interglaziale Moore begannen sich wahrscheinlich um diese Zeit zu bilden, so Schladming, Ampaß und Hopfgarten (Frrbas 1925 und 1927), Wasserburg-Puttenham nach den Großrestuntersuchungen von Schuster (1909) und Pfefferbichl im Allgäu nach eigenen Untersuchungen.

Der Fichten-Tannenzeit folgt eine Kiefernzeit mit hohen Werten von Nichtbaumpollen (vorwiegend Pollen von Cyperaceen). Die Kiefer wird aber bald wieder von der Fichte abgelöst. Gleichzeitig erscheinen noch einmal Tanne und Hainbuche mit geringen Prozentsätzen. Nun folgt eine der ersten sehr ähnliche 


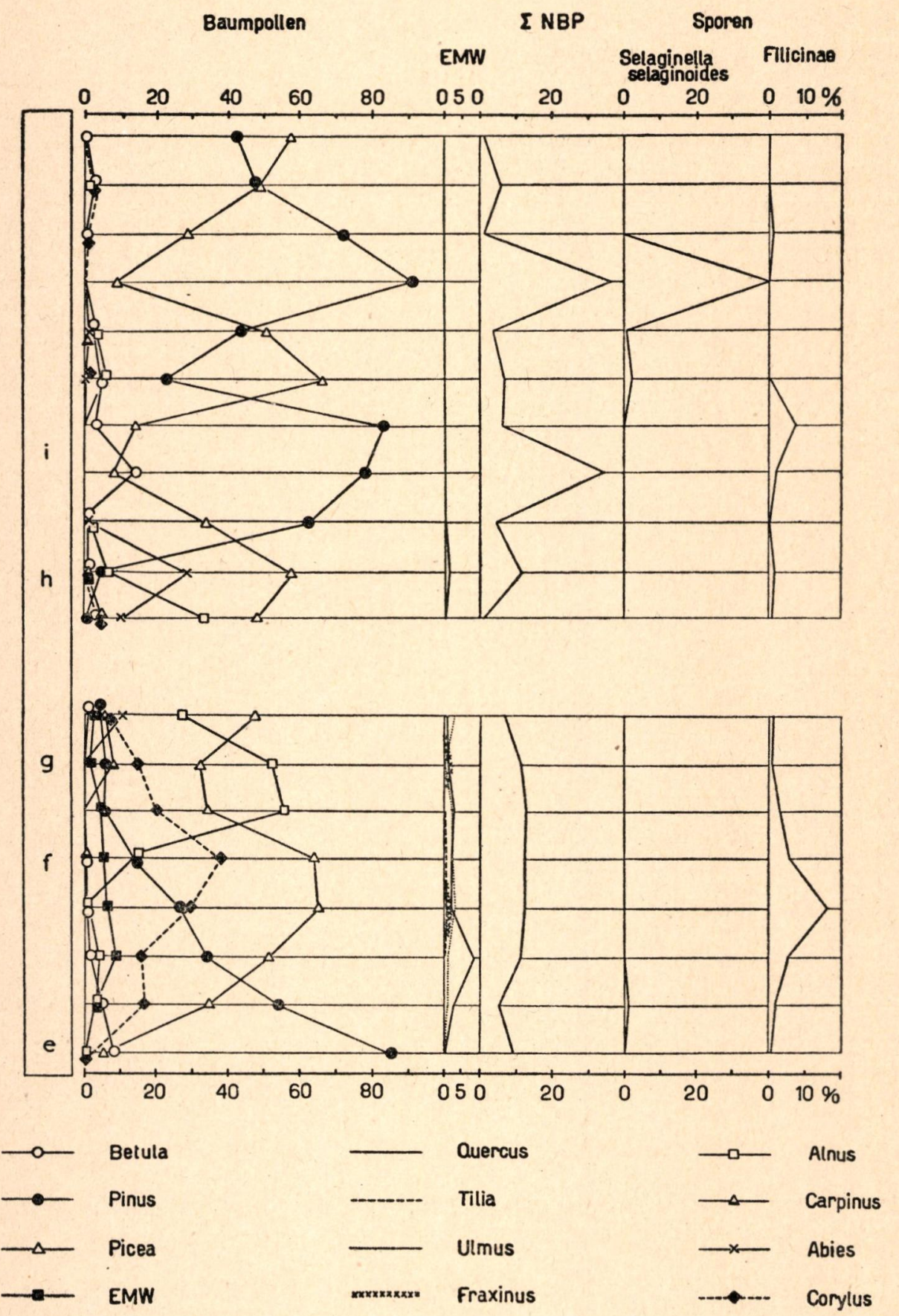

Abb. 1. Interglazial von Großweil. Halbschematisches Pollendiagramm, zusammengestellt aus zwei Profilen: aberer Teil Profil aus den Schieferkohlenflözen, unterer Teil Profil aus den darunter liegenden Schichten. Näheres s. Text. 
zweite Kiefernzeit mit anschließendem erneutem Fichtenanstieg. Damit schließen die pollenführenden Schichten von Großweil ab. Ob sich in Ohlstadt oder Pfefferbichl im Allgäu die Entwicklung noch weiter verfolgen läßt, ist zur Zeit noch ungewiß.

Die Vegetationsentwicklung des Interglazials von Großweil stimmt sehr gut mit der Abfolge der interglazialen Perioden überein, wie sie auf Grund von Pollenuntersuchungen von JESSEN \& MiLthers (1928) in Dänemark, später von verschiedenen Stellen des norddeutschen und polnischen Flachlandes bekannt geworden sind, sehr schön z. B. in Rinnersdorf (vgl. die zusammenfassende Darstellung von WoldstedT 1949). In Abb. 1 wurden die entsprechenden Perioden JEssen's - kleine Buchstaben - am Rande eingetragen. Vor allem finden sich die gegenüber dem Postglazial bezeichnenden Züge wieder: Die späte Lage des Corylusmaximums, das vollständige Fehlen der Rotbuche (Fagus) und ihre Vertretung durch die Hainbuche und die recht späte Ausbreitung der Tanne (Abies). Die starke Beteiligung der Fichte könnte allerdings auch an das vorletzte Interglazial denken lassen (vgl. Woldstedt 1949). Da sich die Fichte im Postglazial vor allem in den Ostalpen und im östlichen Alpenvorland frühzeitig sehr kräftig ausgebreitet hat, ist es jedoch wahrscheinlich, daß gleiche Unterschiede gegenüber dem Flachland schon in den Interglazialzeiten aufgetreten sind und daher nicht ohne weiteres zur Altersbestimmung herangezogen werden dürfen.

Bei den beiden Kiefernzeiten am Ende des Großweiler Interglazials dürfte es sich - darauf deutet auch das Verhalten von Nichtbaumpollen und Selaginella selaginoides hin - um ausgeprägte Phasen der frühglazialen Klimaverschlechterung mit ausgesprochen stadialem Charakter handeln. Ihr Einflußbereich soll u. a. am Interglazial von Pfefferbichl weiter geprüft werden.

Die Mächtigkeit der Schieferkohlenflöze lehrt, daß die in ihrem Vegetationscharakter subarktischen Kiefern- und Fichtenzeiten am Ende des letzten Interglazials bzw. zu Beginn der Würmeiszeit von beträchtlicher Dauer gewesen sind. Utber Versuche zur näheren Bestimmung dieses Zeitraumes soll später berichtet werden. Auf Grund eines Vergleichs mit der Stoffproduktion ähnlicher postglazialer Moore umfaßt er sicher eine Reihe von Jahrtausenden.

Schriftenverzeichnis.

Firbas, F.: Zur Waldentwicklung des Interglazials von Schladming a. d. Enns. - Beih botan. Zentralbl. 41, 295-310. 1925. - Beiträge zur Kenntnis der Schieferkohlen des Inntals und der interglazialen Waldgeschichte der Ostalpen. - Z. f. Gletscherkunde 15, 261-277. 1927. - Die quartäre Vegetationsentwicklung zwischen den Alpen und der Nord- und Ostsee. - Erdkunde 5, 6-15. 1951.

Jessen, K. \& Milthers, V.: Stratigraphical and Palaeontological Studies of Interglacial Freshwater Deposits in Jutland and Northwest Germany. - Danmarks geol. Unders. 2. R. 48, 334-375. 1928.

LüDr, W.: Interglaziale Pollendiagramme aus der Schweiz. - Verh. Schweizer Naturf.Ges. Zürich, 135-137. 1946.

PAUL, H. \& RUoff, S.: Pollenstatistische und stratigraphische Mooruntersuchungen im südlichen Bayern, 1. und 2. Teil. - Ber. bayr. botan. Ges. 19, 84 S. u. 20, 264 S. 1927 u. 1932.

Schuster, J.: Paläobotanische Notizen aus Bayern. - Ber. bayr. botan. Ges. 12. 1909/10.

TroLl, C.: Die jungeiszeitlichen Ablagerungen des Loisach-Vorlandes in Oberbayern. Geol. Rundschau 28, 599-611. 1937.

WoLDSTEDT, P.: Uber die stratigraphische Stellung einiger wichtiger Interglazialbildungen im Randgebiet der nordeuropäischen Vergletscherung. - Z. deutsch. geol. Ges. 99, 96-122. 1949. 